\title{
Single CCTA-Based Universal Biquadratic Filters Employing Minimum Components
}

\author{
Norbert Herencsar, Jaroslav Koton, and Kamil Vrba
}

\begin{abstract}
In this paper a new second-order voltage- and current-mode universal biquadratic frequency filters using only single current conveyor transconductance amplifier (CCTA) and four passive elements are presented. The proposed voltage-mode universal filter has been transformed into current-mode universal filter using adjoint transformation method. Proposed circuits enables to realize the low-, band-, high-pass, band-stop and all-pass responses without changing the circuit topology and the possibility of independent control of the quality factor $Q$ using single passive element. No component matching is required and all the passive and active sensitivities are low. PSPICE simulations and experimental results based on commercially available amplifiers OPA861 and MAX435 are included verify the theoretical conclusions of the proposed circuits.
\end{abstract}

Index Terms - Active filter, adjoint transformation, CCTA, current- and voltage-mode, universal biquadratic filter.

\section{INTRODUCTION}

Frequency filters are linear electric circuits, which are used in wide area of electronics and also are basic building blocks in analogue signal processing. The analogue frequency filters are the most often used in high-speed data communication systems defined by ITU standards, for signal processing in cable modems, in regulation and measurement techniques, in electro acoustics or in automobile industry [1]. In the last decade, the current-mode signal processing circuits have received increasing attention, due to their advantages [2-3] and the highest attention is paid to universal filter realization that can realize all filter functions such as low-, band-, high-pass, band-stop, and all-pass filter response. A number of papers concerning this issue can be found [4-11]. These papers presents voltage- or current-mode universal filters using single CFA (current-feedback amplifier) [4-5], second-generation current conveyor (CCII) [6], second-generation current-controlled conveyor (CCCII) [7], fully-differential second-generation current conveyor (FDCCII) [8], differential-difference current conveyor (DDCC) [9], current differencing buffered amplifier (CDBA) [10] and operational transresistance amplifier (OTRA) [11].

Manuscript received May 28, 2009. The paper has been supported by the Czech Science Foundation project GACR 102/09/1681 and Ministry of Education of the Czech Republic project No. MSM0021630513.

Norbert Herencsar, Jaroslav Koton, and Kamil Vrba are with the Department of Telecommunications, Faculty of Electrical Engineering and Communication, Brno University of Technology, Purkynova 118, 61200 Brno, Czech Republic (phone: +420-541-149-190; fax: +420-541-149-192).
However, the proposed circuits require certain component matching conditions. Hence, the active and passive component sensitivities are larger than unity in absolute value.

In this work, we have proposed a novel voltage-mode universal filter using single current conveyor transconductance amplifier (CCTA) [12] and four passive elements. Using the adjoint transformation method [13] the current-mode frequency filter has been designed. The proposed structures have been simulated using PSPICE and experimentally measured to verify the theoretical analysis. The active and passive sensitivities of the presented structures are low.

\section{DESCRIPTION OF THE CCTA AND FILTER DESIGN}

The current conveyor transconductance amplifier (CCTA) [12] is a combination of the third-generation current conveyor (CCIII) [14] and balanced-output operational transconductance amplifier (BOTA) [15]. In [16] the CCIII in input stage of the CCTA has been replaced by CCCII because of more flexibility in frequency filter and oscillator design. In this work the CCIII has been replaced by CCII [17]. The schematic symbol of the CCTA is shown in Fig. 1.

Relations between the individual terminals of the CCTA can be described by following hybrid matrix:

$$
\left[\begin{array}{c}
i_{\mathrm{y}} \\
v_{\mathrm{x}} \\
i_{\mathrm{z}} \\
i_{\mathrm{o}+} \\
i_{\mathrm{o}-}
\end{array}\right]=\left[\begin{array}{ccccc}
0 & 0 & 0 & 0 & 0 \\
1 & 0 & 0 & 0 & 0 \\
0 & 1 & 0 & 0 & 0 \\
0 & 0 & +g_{\mathrm{m}} & 0 & 0 \\
0 & 0 & -g_{\mathrm{m}} & 0 & 0
\end{array}\right]\left[\begin{array}{c}
v_{\mathrm{y}} \\
i_{\mathrm{x}} \\
v_{\mathrm{z}} \\
v_{\mathrm{o}+} \\
v_{\mathrm{o}-}
\end{array}\right] .(1)
$$

The proposed voltage-mode filter is shown in Fig. 2 (a) employs only one CCTA and four passive elements. The output voltage $\boldsymbol{V}_{o}$ of this circuit is given as follows:

$V_{o}=\frac{s^{2} C_{1} C_{2} V_{i 1}+s C_{2} G_{1} V_{i 2}+G_{2} g_{\mathrm{m}} V_{i 3}-s C_{2} g_{\mathrm{m}} V_{i 4}}{s^{2} C_{1} C_{2}+s C_{2} G_{1}+G_{2} g_{\mathrm{m}}}$

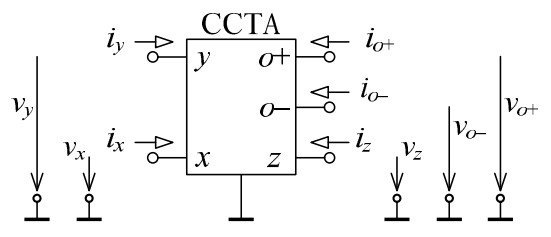

Fig. 1 Schematic symbol of the CCTA 


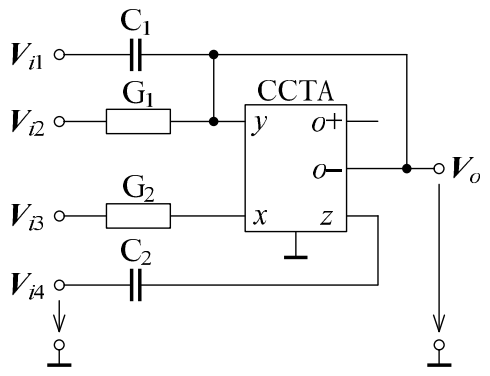

(a)

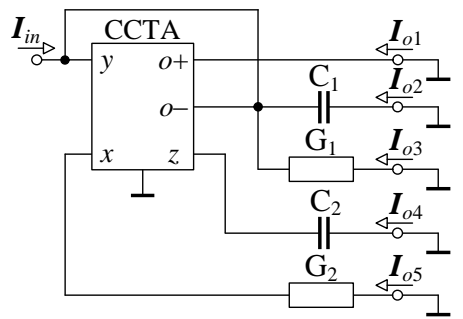

(b)

Fig. 2 (a) The proposed voltage-mode universal filter, (b) the proposed current-mode universal filter

From (2), we can see that:

(i) If $\boldsymbol{V}_{i 2}=\boldsymbol{V}_{i 3}=\boldsymbol{V}_{i 4}=0$ (grounded), a second-order high-pass filter (HP) can be obtained with $\boldsymbol{V}_{o} / \boldsymbol{V}_{i 1}$;

(ii) If $\boldsymbol{V}_{i 1}=\boldsymbol{V}_{i 3}=\boldsymbol{V}_{i 4}=0$ (grounded), a second-order band-pass filter (BP1) can be obtained with $\boldsymbol{V}_{o} / \boldsymbol{V}_{i 2}$;

(iii) If $\boldsymbol{V}_{i 1}=\boldsymbol{V}_{i 2}=\boldsymbol{V}_{i 4}=0$ (grounded), a second-order low-pass filter (LP) can be obtained with $\boldsymbol{V}_{o} / \boldsymbol{V}_{i 3}$;

(iv) If $\boldsymbol{V}_{i 1}=\boldsymbol{V}_{i 2}=\boldsymbol{V}_{i 3}=0$ (grounded), a second-order band-pass filter (BP2) can be obtained with $\boldsymbol{V}_{o} / \boldsymbol{V}_{i 4}$;

(v) If $\boldsymbol{V}_{i 2}=\boldsymbol{V}_{i 4}=0$ (grounded) and $\boldsymbol{V}_{i 1}=\boldsymbol{V}_{i 3}=\boldsymbol{V}_{i n}$, a second-order band-stop filter (BS) can be obtained with $\boldsymbol{V}_{o} / \boldsymbol{V}_{i n}$;

(vi) If $\boldsymbol{V}_{i 2}=0$ (grounded) and $\boldsymbol{V}_{i 1}=\boldsymbol{V}_{i 3}=\boldsymbol{V}_{i 4}=\boldsymbol{V}_{i n}$, a second-order all-pass filter (AP) can be obtained with $\boldsymbol{V}_{\delta} \boldsymbol{V}_{i n}$.

Thus, the circuit is capable of realizing all filter functions. The circuit requires the minimum number of active and passive elements with no requirement for component matching conditions. The proposed voltage-mode universal filter has been transformed into current-mode universal filer using adjoint transformation method [13]. The proposed current-mode filter is shown in Fig. 2 (b). The complex current transfer functions of this circuit with driving current $\boldsymbol{I}_{\text {in }}$ are given as follows:

$$
\begin{aligned}
& \boldsymbol{K}_{\mathrm{I} \_\mathrm{LP}}=\frac{\boldsymbol{I}_{o 1}}{\boldsymbol{I}_{i n}}=-\frac{G_{2} g_{\mathrm{m}}}{\boldsymbol{D}}, \\
& \boldsymbol{K}_{\mathrm{I} \_\mathrm{HP}}=\frac{\boldsymbol{I}_{o 2}}{\boldsymbol{I}_{i n}}=-\frac{\boldsymbol{s}^{2} C_{1} C_{2}}{\boldsymbol{D}}, \\
& \boldsymbol{K}_{\mathrm{I} \_\mathrm{BP} 1}=\frac{\boldsymbol{I}_{o 3}}{\boldsymbol{I}_{i n}}=-\frac{\boldsymbol{s} C_{2} G_{1}}{\boldsymbol{D}}, \\
& \boldsymbol{K}_{\mathrm{I} \_\mathrm{BP} 2}=\frac{\boldsymbol{I}_{o 4}}{\boldsymbol{I}_{i n}}=-\frac{\boldsymbol{s} C_{2} G_{2}}{\boldsymbol{D}}, \\
& \boldsymbol{K}_{\mathrm{I} \_\mathrm{BP} 3}=\frac{\boldsymbol{I}_{o 5}}{\boldsymbol{I}_{i n}}=-\frac{\boldsymbol{s} C_{2} G_{2}}{\boldsymbol{D}},
\end{aligned}
$$

$$
\boldsymbol{K}_{\mathrm{I} \_\mathrm{BS}}=\frac{\boldsymbol{I}_{o 1}+\boldsymbol{I}_{o 2}}{\boldsymbol{I}_{\text {in }}}=-\frac{G_{2} g_{\mathrm{m}}+\boldsymbol{s}^{2} C_{1} C_{2}}{\boldsymbol{D}},
$$

where

$$
\boldsymbol{D}=\boldsymbol{s}^{2} C_{1} C_{2}+s C_{2} G_{1}+G_{2} g_{\mathrm{m}}=0
$$

As seen from (3a)-(3e), the proposed current-mode filter enables low-, band-, and high-pass responses simultaneously. Band-stop filter (3f) can be obtained by adding up the currents $\boldsymbol{I}_{o 1}$ and $\boldsymbol{I}_{o 2}$. If the band-pass responses $\left(\boldsymbol{I}_{o 3}\right.$ or $\boldsymbol{I}_{o 4}$ or $\left.\boldsymbol{I}_{o 5}\right)$ are inverted, than by adding up to $\boldsymbol{I}_{o 1}$ and $\boldsymbol{I}_{o 2}$ all-pass filters can be obtained.

For all filters the characteristic frequency $\omega_{0}$, quality factor $Q$ and bandwidth BW derided from (4) are:

$$
\begin{aligned}
& \omega_{0}=\sqrt{\frac{G_{2} g_{\mathrm{m}}}{C_{1} C_{2}}}, \\
& Q=\frac{1}{G_{1}} \sqrt{\frac{C_{1} G_{2} g_{\mathrm{m}}}{C_{2}}}, \\
& \mathrm{BW}=\frac{\omega_{0}}{Q}=\frac{G_{1}}{C_{1}} .
\end{aligned}
$$

From the equations $(5 a)-(5 c)$ it is evident that the quality factor $Q$ can be controlled independently of characteristic frequency $\omega_{0}$ using passive element $G_{1}$. By replacing appropriate passive element by FET-based voltage-controlled resistor (VCR) [18] the quality factor $Q$ can be controlled electronically, that is particular advantage of the proposed circuits. The characteristic frequency $\omega_{0}$ can be independently adjusted from the bandwidth BW by varying $C_{2}, G_{2}$ or $g_{\mathrm{m}}$ of the proposed frequency filter.

\section{SENSITIVITY ANALYSIS AND NON-IDEAL EFFECTS}

A sensitivity study forms an important index of the performance of any active network. The formal definition of sensitivities [19] is:

$S_{x}^{F}=\frac{x}{F} \frac{\partial F}{\partial x}$

where $F$ represents one of $\omega_{0}, Q, \mathrm{BW}$ and $x$ represents any of the passive elements $\left(C_{1}, C_{2}, G_{1}, G_{2}\right)$ or the active parameter $\left(g_{\mathrm{m}}\right)$. Using the above definition the active and passive sensitivities of the proposed circuits shown in Fig. 2 (a) and Fig. 2 (b) are given as:

$$
\begin{aligned}
& S_{G_{2}, g_{\mathrm{m}}}^{\omega_{0}}=-S_{C_{1}, C_{2}}^{\omega_{0}}=\frac{1}{2}, \quad S_{G_{1}}^{\omega_{0}}=0, \\
& S_{C_{1}, G_{2}, g_{\mathrm{m}}}^{Q}=-S_{C_{2}}^{Q}=\frac{1}{2}, \quad S_{G_{1}}^{Q}=-1, \\
& S_{G_{1}}^{\mathrm{BW}}=-S_{C_{1}}^{\mathrm{BW}}=1, \quad S_{C_{2}, G_{2}, g_{\mathrm{m}}}^{\mathrm{BW}}=0 .
\end{aligned}
$$

From the results it is evident that the sensitivities are low and not larger than unity in absolute value.

Taking into account the non-ideal CCTA, namely $i_{\mathrm{y}}=0$, 
$v_{\mathrm{x}}=\beta v_{\mathrm{y}}, \quad i_{\mathrm{z}}=\alpha i_{\mathrm{x}}, \quad i_{\mathrm{o}+}=+g_{\mathrm{m}} v_{\mathrm{z}}, \quad$ and $\quad i_{\mathrm{o}-}=-g_{\mathrm{m}} v_{\mathrm{z}}, \quad$ where $\alpha=1-\varepsilon_{\mathrm{i}}$ and $\varepsilon_{\mathrm{i}}\left(\left|\varepsilon_{\mathrm{i}}\right| \quad 1\right)$ is the current tracking error from $x$ terminal to $z$ terminal, $\beta=1-\varepsilon_{\mathrm{v}}$ and $\varepsilon_{\mathrm{v}}\left(\left|\varepsilon_{\mathrm{v}}\right| \quad 1\right)$ is the input voltage tracking error from $x$ terminal to $y$ terminal of CCTA, respectively. The transconductance $g_{\mathrm{m}}$ of the BOTA with the non-idealities can be assumed as [20]:

$$
g_{\mathrm{m}}=\frac{g_{\mathrm{m}} \omega_{g}}{s+\omega_{g}} \cong g_{\mathrm{m}}(1-\mu s)
$$

where $\omega_{g}$ is the first-pole of the BOTA and $\mu=1 / \omega_{g}$. Taking into account the non-idealities of the CCTA mentioned above, the denominator of the transfer functions (4) becomes:

$$
\boldsymbol{D}=\boldsymbol{s}^{2} C_{1} C_{2}+s C_{2} G_{1}\left(1-\frac{\alpha \beta G_{2} g_{\mathrm{m}} \mu}{C_{2} G_{1}}\right)+\alpha \beta G_{2} g_{\mathrm{m}}=0
$$

Due to the parasitic effect, the characteristic departs from the ideal responses. But, the parasitic effect can be made negligible satisfying the following condition:

$$
\frac{\alpha \beta G_{2} g_{\mathrm{m}} \mu}{C_{2} G_{1}} \quad 1
$$

\section{SiMUlATION AND EXPERIMENTAL RESUltS}

The behaviour of the proposed voltage- and current-mode universal filter in Fig. 2 (a)-(b) has been verified using PSPICE simulations. Used internal structure of the CCTA is shown in Fig. 3. The CCII+ is formed by transistors $Q_{1}-Q_{17}$ and the BOTA consists of transistors $Q_{18}-Q_{29}$. In the design the transistor model parameters NR100N (NPN) and PR100N (PNP) of bipolar arrays ALA400 from AT\&T [21] were used. Bias current $I_{\mathrm{O}}=400 \mu \mathrm{A}$ has been chosen. The transconductance $g_{\mathrm{m}}$ of CCTA can be adjusted by current $I_{\mathrm{B}}=g_{\mathrm{m}} / 20$.

For practical measurement the CCTA has been realized by the structure shown in Fig. 4. Commercially available circuits OPA861 and MAX435 have been used. The measurement was carried out with an Agilent 4395A network/spectrum analyzer. For the characteristic frequency $f_{0}=\omega_{0} / 2 \pi \cong 1 \mathrm{MHz}$ and the quality factor of filters $Q=1$ the following passive component values have been chosen: $C_{1}=C_{2}=150 \mathrm{pF}, \quad G_{1}=G_{2}=1 \mathrm{mS} \quad\left(R_{1}=R_{2}=1 \mathrm{k} \Omega\right) \quad$ and $g_{\mathrm{m}}=1 \mathrm{mS}\left(I_{\mathrm{B}}=50 \mu \mathrm{A}\right)$.

The simulation and measurement results of the low-, band-, and high-pass filter working in voltage-mode are shown in Fig. 5 (a). It is evident that the measured responses are in agreement with the simulations. In the higher-frequency region the real properties of the OPA861, MAX435 circuits and parasitic capacities or inductances of the constructed prototypes start to be significant. The simulation results of the low-, band-, and high-pass filter working in current-mode are shown in Fig. 5 (b).

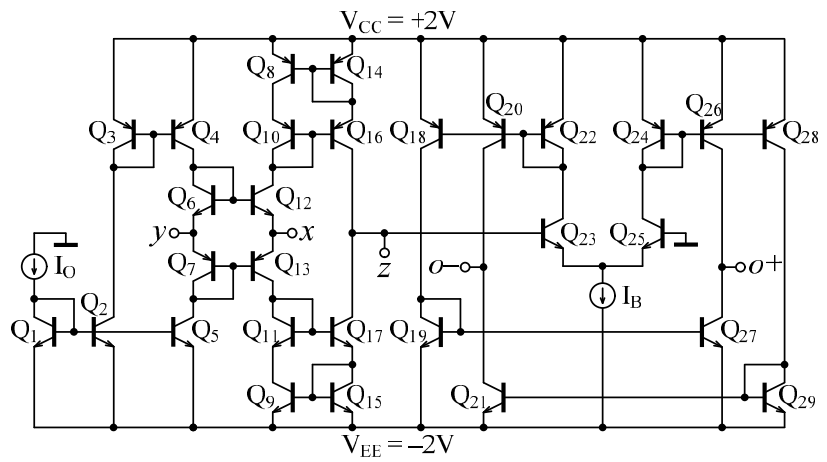

Fig. 3 Used bipolar implementation of the CCTA

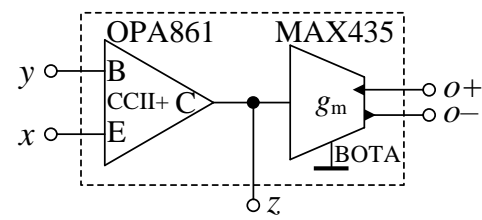

Fig. 4 Possible realization of the CCTA using plus-type CCII (OPA861) and balanced-output OTA (MAX435)

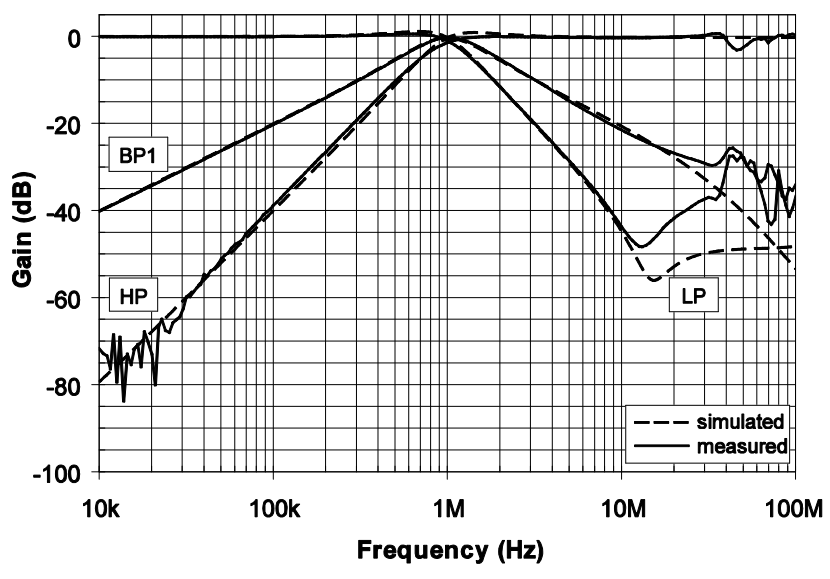

(a)

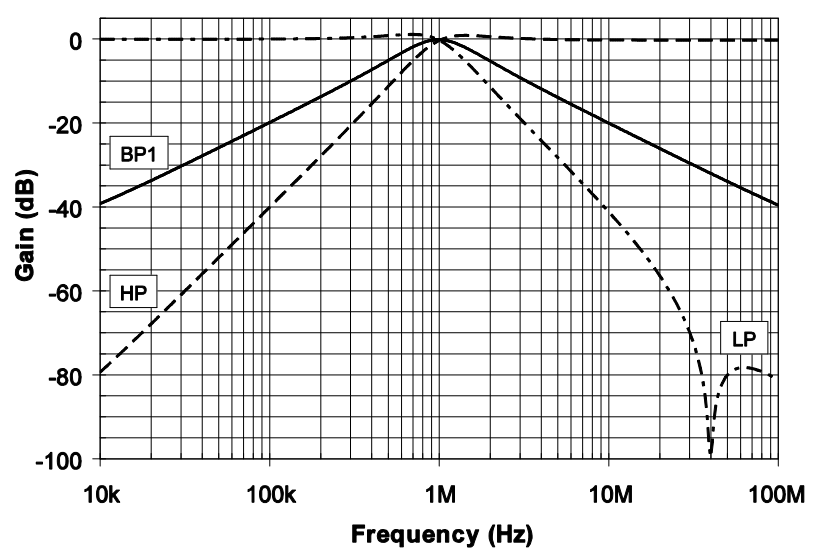

(b)

Fig. 5 (a) Simulated and measured frequency characteristics for LP, BP1, and HP responses of the proposed circuit of Fig. 2 (a) in voltage mode, (b) simulated frequency characteristics for LP, BP1, and HP responses of the proposed circuit of Fig. 2 (b) in current mode

\section{CONClusion}

A new voltage- and current-mode universal frequency 
filters using single CCTA and four passive elements are presented. All passive elements are virtually grounded in the structure. The use of only grounded capacitors is ideal for integrated circuit implementation [22]. The current-mode filter has been obtained using adjoint transformation from the voltage-mode filter. Both structures enable to realize the low-, band-, high-pass, band-stop and all-pass response without changing the circuit topology. The independent control of the quality factor $Q$ using single passive element is possible, which can be advantageous in some applications. The behaviours of the proposed filter have been verified by PSPICE simulations. Corresponding bipolar implementation of the CCTA has been presented. The behaviours of the voltage-mode structure have been furthermore verified by experimental measurements.

\section{REFERENCES}

[1] G. Ferri and N. C. Guerrini, Low-Voltage Low-Power CMOS Current Conveyors. London: Kluwer Publ., 2003.

[2] C. Toumazou, F. J. Lidgey, and D. G. Haigh, Analogue IC Design: The current-mode approach. London: Peter Peregrinus Ltd., 1990.

[3] S. Takagi, "Analog circuit designs in the last decade and their trends toward the 21st century," IEICE Trans. Fundamentals, vol. E84-A, pp. $68-79,2001$

[4] J. W. Horng, C. K. Chang, and J. M. Chu, "Voltage-mode universal biquadratic filter using single current-feedback amplifier," IEICE Trans. Fundamentals, vol. E85-A, no. 8, pp. 1970-1973, 2002.

[5] R. K. Sharma and R. Senani, "Universal current mode biquad using a single CFOA," Int. Journal of Electronics, vol. 91, pp. 175-183, 2004.

[6] M. Sagbas and M. Koksal, "Voltage-mode three-input single-output multifunction filters employing minimum number of components," Frequenz, vol. 61, pp. 87-93, 2007

[7] C. M. Chang, T. H. Huang, S. H. Tu, C. L. Hou, and J. W. Horng, "Universal active current filter using single second-generation current controlled conveyor," Int. J. of Circ., Syst. and Sign. Proc., vol. 1, pp. 194-198, 2007.

[8] C. M. Chang and H.P. Chen, "Single FDCCII-based tunable universal voltage-mode filter," Circ. Syst. Sign. Proc., vol. 24, pp. 221-227, 2005.

[9] H. P. Chen and K. H. Wu, "Single DDCC-based voltage-mode multifunction filter," IEICE Trans. Fundamentals, vol. E90-A, pp. 2029-2031, 2007.

[10] A. Ü. Keskin, "Multi-function biquad using single CDBA," Electrical Engineering, vol. 88, pp. 353-356, 2006.

[11] S. K1lınç, A. Ü. Keskin, and U. Cam, "Cascadable voltage-mode multifunction biquad employing single OTRA," Frequenz, vol. 61, pp 84-86, 2007.

[12] R. Prokop and V. Musil, "Modular approach to design of modern circuit blocks for current signal processing and new device CCTA," in Proc. 7th IASTED SIP'05, Hawaii, USA, pp. 494-499, 2005.

[13] T. Dostal, D. Biolek, and K. Vrba, "Adjoint voltage-current mode transformation for circuits based on modern current conveyors," in Proc. 4th IEEE Int. Caracas Conf. ICCDCS'02, Aruba, pp. T034-1-4, 2002.

[14] A. Fabre, "Third-generation current conveyor: a new helpful active element," Electronics Letters, vol. 31, pp. 338-339, 1995.

[15] R. L. Geiger and E. Sanchez-Sinencio, "Active Filter Design Using Operational Transconductance Amplifiers: A Tutorial,” IEEE Circuits and Devices Magazine, vol. 1, pp. 20-32, 1985.

[16] M. Siripruchyanun and W. Jaikla, "Current controlled current conveyor transconductance amplifier (CCCCTA): a building block for analog signal processing," Electrical Engineering, vol. 90, pp. $443-453,2008$

[17] A. Sedra and K. C. Smith, "A second generation current conveyor and its applications," IEEE Trans. Circ. Theory, vol.17, pp.132-134, 1970.

[18] R. Senani, "Realization of linear voltage-controlled-resistance in floating form," Electronics Letters, vol. 30, pp. 1909-1911, 1994.

[19] W. K. Chen, The Circuits and Filters Handbook. New York: CRC Press, 2003.

[20] H. P. Chen, S. S. Shen, and J.P. Wang, "Electronically tunable versatile voltage-mode universal filter," Int. J. Electron. Commun. (AEU), vol. 62, pp. 316-319, 2008 .
[21] D. R. Frey, "Log-domain filtering: an approach to current-mode filtering," IEE Proc. G, Circ. Dev. Syst., vol. 140, pp. 406-416, 1993.

[22] M. Bhusan and R. W. Newcomb, "Grounding of capacitors in integrated circuits," Electronics Letters, vol. 3, pp. 148-149, 1967.

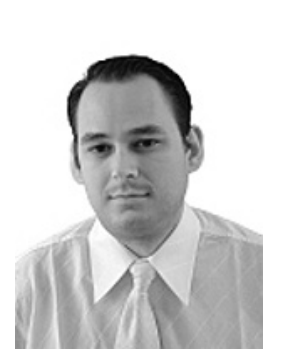

Norbert Herencsar received the Master of Science (M.Sc.) degree in Electrical Engineering from Brno University of Technology, Czech Republic, in 2006. Currently, he is an Assistant Professor and a Ph.D. student at the Department of Telecommunications, Faculty of Electrical Engineering and Communication, Brno University of Technology, Brno, Czech Republic. His research interests include analog signal processing, circuit theory and practice, new active elements and their circuit applications, and oscillators. He has infused novel active elements for analog signal processing such as Current Follower Transconductance Amplifier and Differential-input Buffered and Transconductance Amplifier. He is an author or co-author of about 30 research articles published in international journals or conference proceedings.

Mr. Herencsar is Student Member of IEEE and Member of IACSIT, IAENG, and ACEEE.

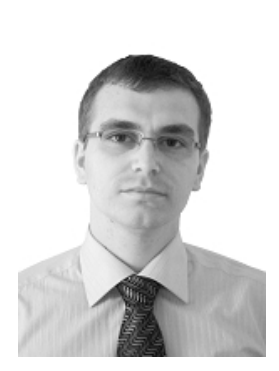

Jaroslav Koton received the M.Sc. and Ph.D. degree in Electrical Engineering from Brno University of Technology in 2005 and 2009, respectively. Currently he works as Assistant Professor at the Department of Telecommunications of the Faculty of Electrical Engineering and Communication of Brno University of Technology, Czech Republic. He is focused on linear-circuits designing methods with current or voltage conveyors, and current active elements. He is an author or co-author of about 45 research articles published in international journals or conference proceedings.

Dr. Koton is Member of IEEE and IACSIT.

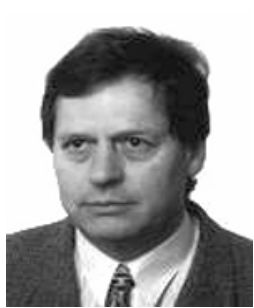

Kamil Vrba received the Ph.D. degree in Electrical Engineering in 1976, and the Prof. degree in 1997, both from the Technical University of Brno. Since 1990 he has been Head of the Dept. of Telecommunications, Faculty of Electrical Engineering and Computer Science, Brno University of Technology, Brno, Czech Republic. His research work is concentrated on problems concerned with accuracy of analog circuits and mutual conversion of analog and digital signals. In cooperation with AMI Semiconductor (now ON Semiconductor) he has developed number of novel active function blocks for analog signal processing such as Universal Current Conveyor, Universal Voltage Conveyor, Programmable Current Amplifier, and others. He is an author or co-author of more than 600 research articles published in international journals or conference proceedings.

Prof. Vrba is Associate Member of IET and Member of IEEE and IEICE. 